\title{
Using PF-MOUTH GELTM for Sore or Painful Tongue Improved Symptoms and Stabilized Dryness and Trapping of Food: A Case Report
}

Shuichi Tsubura, PhD, DDSc ${ }^{1,2 *}$; Masahide Kanazawa, PhD, DDSc ${ }^{3}$; Shunya Oka, PhD ${ }^{4}$; Rika Hirama, DH².TsutomuTsubura, PhD, DDSc

'Department of Biochemistry, Nippon Dental University School of Life Dentistry at Niigata, Hamaura-cho, Chuo-ku, Niigata 95 I-8580, Japan

${ }^{2}$ Tsubura Dental Clinic, 9-6 Enomachi, Utsunomiya,Tochigi 320-0802, Japan

${ }^{3}$ Kanazawa Dental Clinic, 500-4 Harigaya-machi, Utsunomiya, Tochigi 32 I-01 47, Japan

${ }^{4}$ Department of Biology, Nippon Dental University School of Life Dentistry at Niigata, Hamaura-cho, Chuo-ku, Niigata 95 I-8580, Japan

\section{"Corresponding author}

Shuichi Tsubura, PhD, DDSc,

Department of Biochemistry, Nippon Dental University School of Life Dentistry at Niigata, Hamaura-cho, Chuo-ku, Niigata 95 I-8580, Japan;Tel. +8I-25-267-I500; Fax:+8I-25-267-I I34; E-mail: tshu@ucatv.ne.jp

\section{Article information}

Received: Feburary $I^{\text {st }}, 2021$; Revised: February $22^{\text {nd }}, 2021$; Accepted: February $24^{\text {th }}, 2021$; Published: February $24^{\text {th }}, 2021$

\section{Cite this article}

Tsubura S, Kanazawa M, Oka S, Hirama R, Tsubura T. Using PF-MOUTH GELTM for sore or painful tongue improved symptoms and stabilized dryness and trapping of food:A case report. Dent Open J. 202I; 7(I): 7-II. doi: 10.17I40/DOJ-7-I43

\section{ABSTRACT}

We describe herein the case of a 72-year-old woman with persistent and symptomatic sore or painful tongue (SPT) treated using PF-MOUTH GEL TM (PF-Gel; Daiichi-Sangyo, Osaka, Japan), which includes 30\% fucoidan and 0.75\% sword bean. PF-MOUTH GEL ${ }^{\text {TM }}$ was applied to the tongue and kept in place for 3-min. Application was performed twice a day (morning and evening) for 3-months, and resulted in marked improvement of symptomatic sore or painful tongue. Because only one case was reported in this study, clinical trials are required to confirm the efficacy and safety of topical PF-MOUTH GEL ${ }^{\text {TM }}$ for the treatment of symptomatic sore or painful tongue.

\section{Keywords}

Tongue; Fucoidan; Inflammation; Treatment; Gel.

\section{INTRODUCTION}

$\mathrm{T}$ he clinical characteristics of sore or painful tongue (SPT) are well-defined and often involve atrophic glossitis, fissured tongue, median rhomboid glossitis, or burning tongue, although the precise etiology remains unclear or incomplete and definitive treatment has yet to be clarified. The diagnosis, clinical presentation and severity of symptomatic sore or painful tongue are key factors in selecting treatment. The objectives of treatment are controlling pain, suppressing the inflammatory response, and improving quality of life for the patient. Drug delivery represents a major challenge, because topical medications are easily rubbed or rinsed away from the target area through normal oral movements and salivary flow. The use of topical corticosteroid ointments for patients with symptomatic sore or painful tongue is expected to control the inflammatory process associated with the formation of symptomatic sore or painful tongue, but side effects such as burning, changes in taste perception and secondary oral candidiasis may be induced.
Some effectiveness of corticosteroid ointment has been shown in the treatment of symptomatic sore or painful tongue, but has not been satisfactory. Furthermore, treatment for one month or more has been required to reduce symptoms.

Fucoidans are fucose-rich polymers that were identified in brown algae by Kylin in 1918. ${ }^{1}$ Fucoidans have been reported to show bio-activities ${ }^{2-4}$ such as anti-viral, anti-bacterial, anti-coagulant and anti-tumoral properties. Further, fucoidans have been used as supplements in cancer patients, and reportedly exert anti-inflammatory effects in patients with advanced cancer. ${ }^{5}$ Although, many studies have attempted to determine the effective of fucoidans as medicines or cosmetics, few have examined effects on oral diseases.

In a previous case study, we reported that Power Fucoidan Cream (PFC; Daiichi Sangyo, Osaka, Japan) comprising 4\% 
fucoidan isolated from Nemacystus decipiens, achieved marked improvement of recurrent aphthous stomatitis, ${ }^{6}$ and oral herpes labialis. ${ }^{7}$ We have also reported ${ }^{8}$ that fucoidans showed anti-microbial activity against Streptococcus mutans, and significantly inhibited the adhesion of $S$. mutans to bovine teeth and porcelain. PF-MOUTH GEL ${ }^{\text {TM }}$ (PF-Gel; Daiichi-Sangyo) includes 30\% fucoidans and $0.75 \%$ sword bean (Figure 1), and has been developed to overcome weaknesses of of Power Fucoidan Cream ${ }^{\mathrm{TM}}$ such as difficulty retaining the cream on mucous membranes and a bitter taste. In this report, we describe a case of painful symptomatic sore or painful tongue that proved resistant to various medications. This case was successfully treated using topical application of PF-MOUTH GEL $^{\text {TM }}$ (Figure 1). This is the first report describing topical use of PF-MOUTH GEL ${ }^{\mathrm{TM}}$ on symptomatic sore or painful tongue.

\section{CASE REPORT}

A 72-year-old Japanese woman with painful symptomatic sore or painful tongue visited our clinic. At 58-years-old, she had been diagnosed with chronic illnesses such as hypertension and hyperlipoidemia, and subsequently developed symptomatic sore or painful tongue. Various medications including topical corticosteroid ointment (triamcinolone acetonide) and non-steroidal antiinflammatory drugs had been attempted, but the lesions proved resistant to all treatments. Dermatological and clinical examination revealed fissured tongue, with deep grooves on the surface and annular white plaques on the middle part of the tongue (Figure 2), and the superficial mucosa of the tongue was red and uneven. The patient was constantly suffering from sores and pain on the tongue, making eating and drinking difficult. The clinical evaluation suggested that, it was not a purulent tongue inflammation. In addition, this case is not an infectious tongue lesion, as blood test values are stable during the clinical trial (Table 1).

After obtaining informed consent, PF-MOUTH GEL ${ }^{\mathrm{TM}}$ was applied to the tongue after tooth brushing and kept in place for 3-min. PF-MOUTH GEL ${ }^{\mathrm{TM}}$ was applied to the surface of the tongue with light force by the patient using her fingertips. Application was preformed twice a day, in the morning and evening (Figure $3 \mathrm{~A}$ ). Treatment was continued for 3-months. At 1 -month after starting treatment, deep grooves were still visible on the surface of the tongue, but the number of grooves had decreased. The color tone of the tongue surface had improved from bright red to coral. Symptoms of soreness, pain, and trapping had slightly improved without any side effects (Figure 3B, Table 1) and no exacerbation of any symptoms. After 3-months, deep grooves in the tongue surface were further decreased (Figure 3C, 3D; Table 1).

Follow-ups at 4-months (Figure 3E, Table 1), 5-months

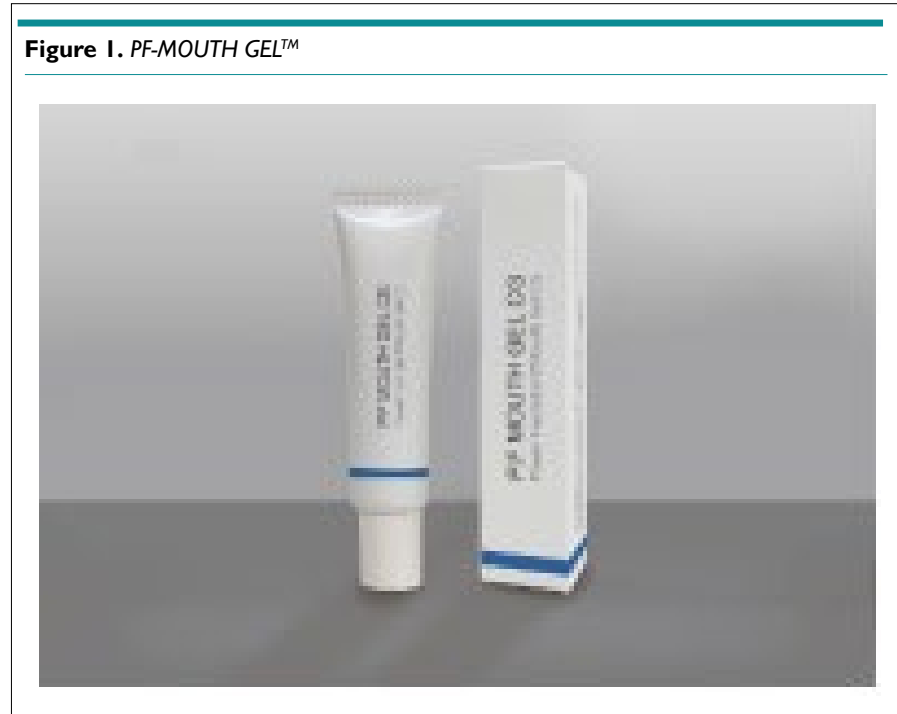

Figure 2. Application of PF-MOUTH GEL ${ }^{T M}$ to the Patient's Tongue (Before status)

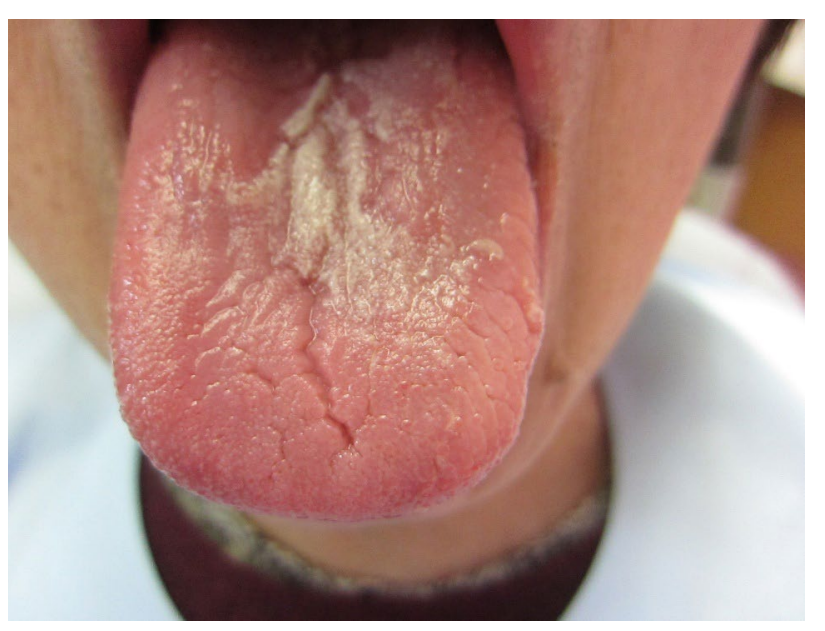

\begin{tabular}{|c|c|c|c|c|c|c|}
\hline & WBC $\left(10^{2} / \mu L\right)$ & $\mathrm{Hb}(\mathrm{g} / \mathrm{dL})$ & Ht (\%) & HDL (mg/dL) & LDL (mg/dL) & T-G (mg/dL) \\
\hline Before treatment & 69 & 14.0 & 41.6 & 75 & 105 & 83 \\
\hline After I-month & 69 & 14.0 & 41.6 & 75 & 105 & 83 \\
\hline 2-months & 59 & 14.1 & 43.4 & 74 & 127 & 100 \\
\hline 3-months & 65 & 14.0 & 42.2 & 73 & 125 & 100 \\
\hline 4-months & 62 & 14.6 & 44.1 & 76 & 120 & 103 \\
\hline 5-months & 67 & 13.8 & 43.3 & 68 & 109 & 102 \\
\hline 6-months & 62 & 14.2 & 45.3 & 71 & 113 & 113 \\
\hline I2-months & 71 & 13.6 & 42.3 & 62 & 108 & 109 \\
\hline \multicolumn{7}{|c|}{ 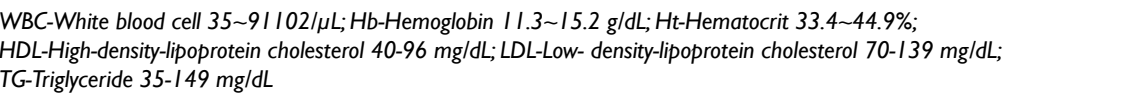 } \\
\hline
\end{tabular}




\section{Openventio}

Figure 3

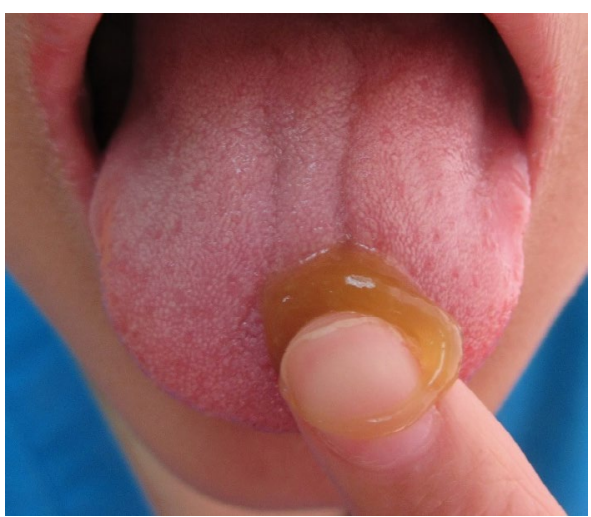

3A. Applying PF-MOUTH GEL ${ }^{T M}$ (Subject Different from the Case)

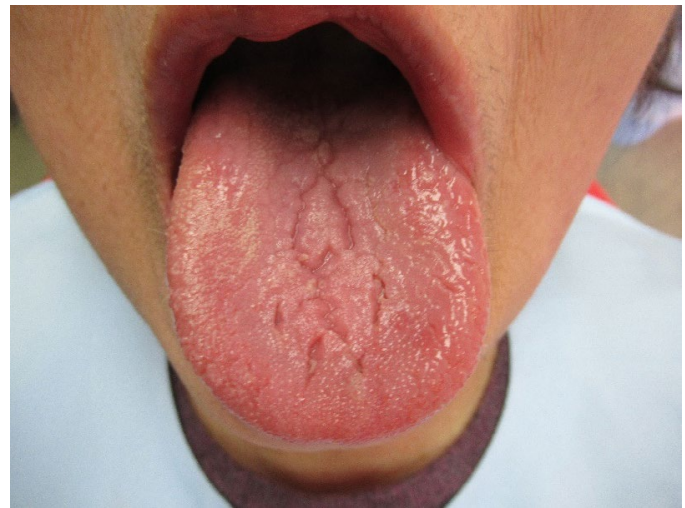

3C. 2-months later

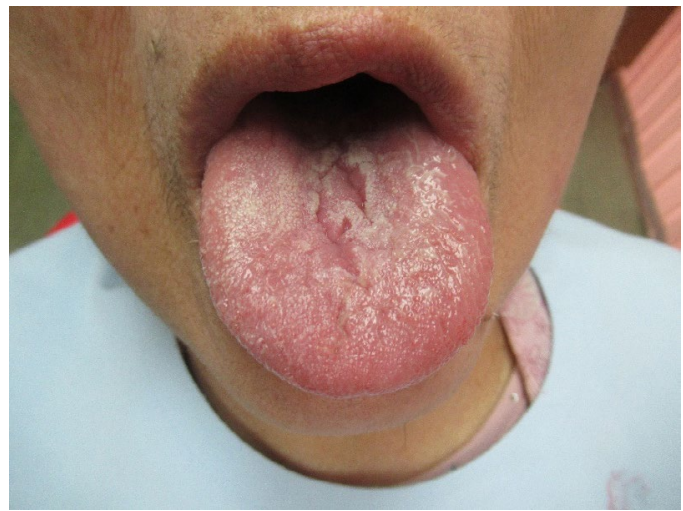

3E. 4-months later

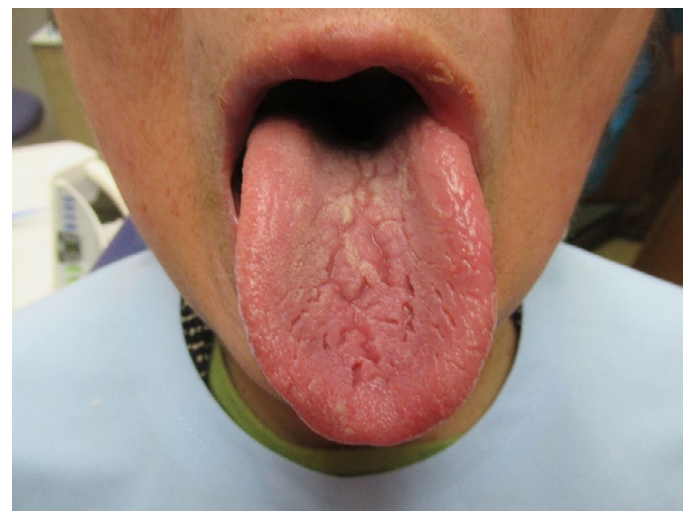

3G. 6-months later

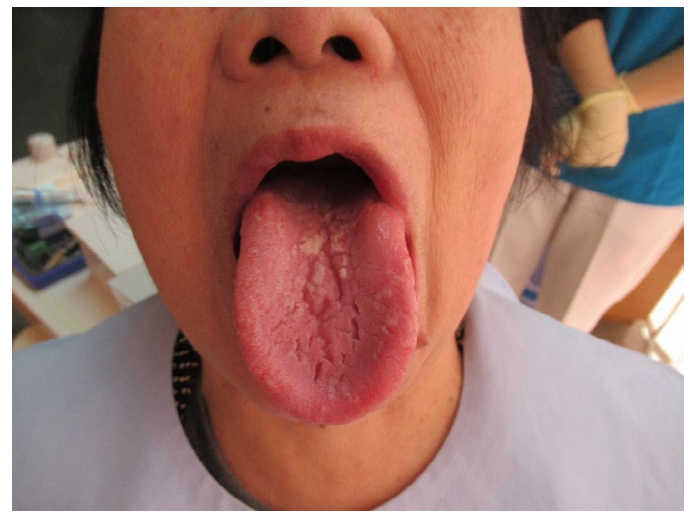

3B. I-month later

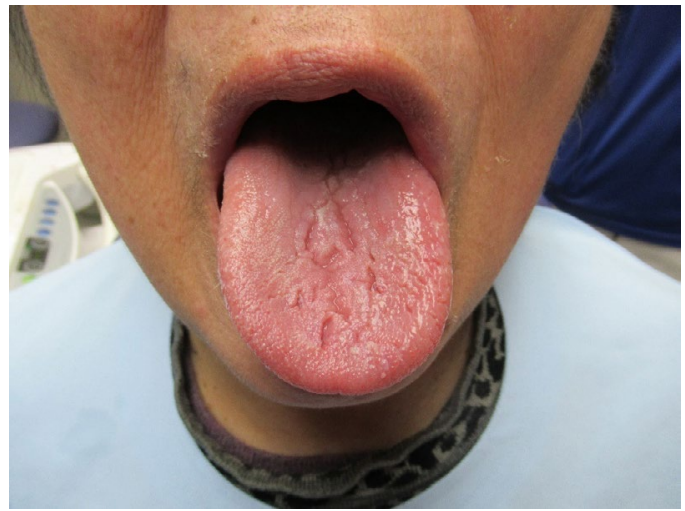

3D. 3-months later

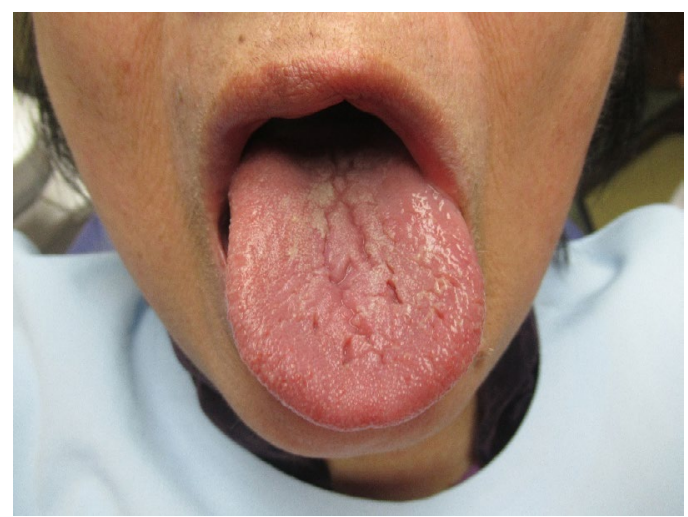

3F. 5-months later

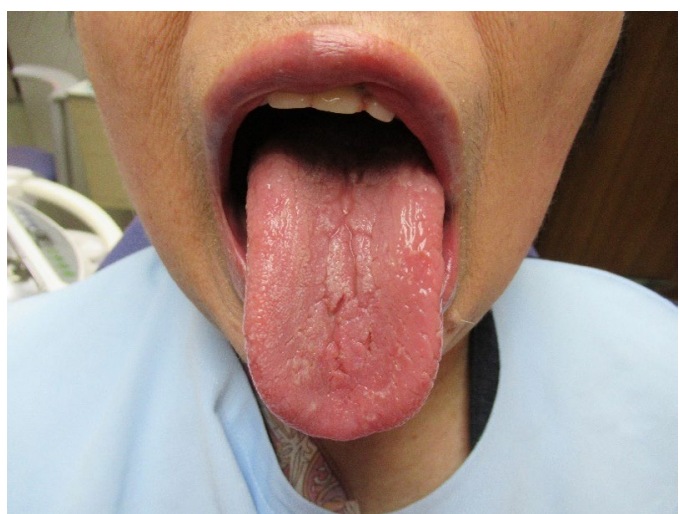

3H. 12-months later 


\begin{tabular}{|c|c|c|c|c|c|c|c|c|}
\hline & BT & I-month & 2-month & 3-month & 4-month & 5-month & 6-month & 12-month \\
\hline Soreness & I & 2 & 2 & 3 & 3 & 3 & 3 & 3 \\
\hline Pain & I & 2 & 3 & 3 & 3 & 3 & 3 & 3 \\
\hline Malodor & I & I & 2 & 2 & 2 & 2 & 2 & 2 \\
\hline Dryness & I & I & I & 2 & 2 & 2 & 2 & 2 \\
\hline Trapping & I & 2 & 3 & 3 & 3 & 3 & 3 & 3 \\
\hline
\end{tabular}

(Figure 3F, Table 1), 6-months (Figure 3G, Table 1), and 12-months (Figure $3 \mathrm{H}$, Table 1) revealed no esthetic or clinical problems. Deep grooves on the tongue surface and annular white plaques had decreased. As of the last follow-up, tongue symptoms had almost completely resolved. No systemic effects of PF-MOUTH GEL ${ }^{\text {TM }}$ during daily life were identified (Table 2).

\section{DISCUSSION}

Symptomatic sore or painful tongue is a mucous inflammatory condition of unknown etiology. Typical clinical conditions in symptomatic sore or painful tongue include atrophic glossitis, fissured tongue, and burning tongue. The patient in this case complained of highly painful lesions and trouble with food intake for several years. Tongue problems included a variety of signs and symptoms, such as soreness or pain, color changes, and changes in taste perception. Diagnosis of tongue abnormalities requires examination of tongue morphology, thorough elicitation of a detailed history, symptom durations and intra-oral lifestyle habits, such as use of tobacco products or intake of alcoholic beverages. The 1988-1994 National Health and Nutrition Examination Survey described the prevalence of tongue lesions as $15.5 \%$ among adults in the United States. The most common abnormal condition of the tongue is geographic tongue, followed by fissured tongue and hairy tongue. ${ }^{9}$ Our patient was instructed to dab PF-MOUTH GEL ${ }^{\text {TM }}$ on the affected areas of the slightly dried tongue to keep the gel in place for 3-min, and to avoid eating or drinking for 30-min after application. PF-MOUTH GEL ${ }^{\text {TM }}$ contains 30\% fucoidan and $0.75 \%$ sword bean extract, along with propylene glycol (PG), glycerin, polyethylene glycol 8 (PEG-8), xylitol, xanthan gum and water. The fucoidan is extracted from $N$. decipiens from Tonga and sword bean grown in Japan.

In this case, we diagnosed the tongue condition as fissured tongue or burning tongue based on clinical circumstantial evidence, although biopsy was not performed. Siddhanta and Murthy ${ }^{10}$ reported that fucoidan showed anti-tumoral and antiinflammatory effects, and Aisa et $\mathrm{a}^{11}$ found that fucoidan exhibits anti-cancer effects, such as against human lymphoma HS-Sultan cells. According to such activities, fucoidan appears to have pharmaceutical potential in various applications, such as for anti-viral medication and cancer therapy.

Various medications have been applied for symptomatic sore or painful tongue, but consistent good results have remained elusive. In the previous, we have experienced significant cases using Power Fucoidan Cream ${ }^{\mathrm{TM}}$ on the lip mucosa. ${ }^{6,7}$ However, it was considered that the Power Fucoidan Cream ${ }^{\mathrm{TM}}$ was not optimal for the surface layer of the tongue due to insufficient viscosity. We therefore decided to apply PF-MOUTH GEL ${ }^{\mathrm{TM}}$. During applications of PF-MOUTH GEL ${ }^{\mathrm{TM}}$, the patient experienced no side effects and did not complain of stinging. The most dramatic effect was the rapidity of healing for sore and painful lesions. Such activity may occur via the inhibition of enzymes including matrix metalloproteinases, hyaluronidases and elastases. ${ }^{12}$

This report is the first to describe topical use of PFMOUTH GEL ${ }^{\text {TM }}$ for symptomatic sore or painful tongue, and further studies are required to determine the effectiveness and safety of topical PF-MOUTH GEL ${ }^{\mathrm{TM}}$ for symptomatic sore or painful tongue.

\section{ACKNOWLEDGMENTS}

The authors wish to thank Dr. Hiromi Shimomura for the helpful and critical discussions.

\section{AUTHOR CONTRIBUTIONS}

All authors contributed equally to this work. S. Tsubura designed the study and interpreted the results. M. Kanazawa, S. Oka, R. Hirama and $\mathrm{T}$. Tsubura collected test data and drafted the manuscript.

\section{CONFLICTS OF INTEREST}

The authors declare that they have no conflicts of interest.

\section{REFERENCES}

1. Kylin H. Biochemistry of seaweeds. Z Physiol Chem. 1918; 101: 236-245.

2. Berteau O, Mulloy B. Sulfated fucans, fresh perspectives: structures, functions, and biological properties of sulfated fucans and an overview of enzymes active toward this class of polysaccharide. Glycobiology. 2003; 13: 29-40. doi: 10.1093/glycob/cwg058

3. Ribeiro AC, Vieira RP, Mourao PAS, Muloy B. A sulfated alpha-L-fucan from sea cucumber. Carbohydr Res. 1994; 255: 225-240. doi: 10.1016/s0008-6215(00)90981-9 
4. Teruya K, Kusumoto Y, Eto H, Nakamichi N, Shirahata S. Selective suppression of cell growth and programmed cell death-ligand 1 expression in HT 1080 fibrosarcoma cells by low molecular weight fucoidan extract. Mar Drugs. 2019; 17(7): 421. doi: 10.3390/ md17070421

5. Takahashi H, Kawaguchi M, Kitamura K, Narumiya S, Kawamura M, Tengan I, et al. An exploratory study on the anti-inflammatory effects of fucoidan in relation to quality of life in advanced cancer patients. Integr Cancer Ther. 2018; 17(2): 282-291. doi: $10.1177 / 1534735417692097$

6. Tsubura S, Waki Y, Tsubura T. Recurrent aphthous stomatitis treated with fucoidan. J Cranio-Maxillary Dis. 2012; 1(2): 105-108. doi: 10.4103/2278-9588.105699

7. Tsubura S, Suzuki A. Case report using 4\% fucoidan cream for recurrent oral herpes labialis: Patient symptoms markedly improved in terms of time to healing and time to loss of discomfort. Dent Open J. 2017; 5(1): 19-13. doi: 10.17140/DOJ-5-135

8. Oka S, Okabe M, Tsubura S, Mikami M, Imai A. Properties of fucoidans beneficial to oral healthcare. Odontology. 2020; 108(1): 34-
43. doi: 10.1007/s10266-019-00437-3

9. Shulman JD, Beach MM, Rivera-Hidalgo F. The prevalence of oral mucosal lesions in U.S. adults: Data from the Third National Health and Nutrition Examination Survey, 1988-1994. J Am Dent Assoc. 2004; 135(9): 1279-1286. doi: 10.14219/jada.archive.2004.0403

10. Siddhanta AK, Murthy ASK. Bioactive polysaccharides from marine brown algae (Phaeophyceae). J Indian Chem Soc. 2001; 78: 431 437.

11. Aisa Y. Fucoidan induces apoptosis of human HS-sultan cells accompanied by activation of caspase-3 and down-regulation of ERK pathways. Am J Hematol. 2005; 78: 7-14. doi: 10.1002/ ajh.20182

12. Moon HJ, Lee SH, Ku MJ, Yu BC, Jeon MJ, Jeong SH, et al. Fucoidan inhibits UVB-induced MMP-1 promoter expression and down regulation of type I procollagen synthesis in human skin fibroblasts. Eur J Dermatol. 2009; 19: 129-134. doi: 10.1684/ ejd.2008.0611 